\title{
Performative prototyping in collaborative mixed reality environments: an embodied design method for ideation and development in virtual reality.
}

\author{
Joris Weijdom \\ 1. Human Media Interaction, University of Twente, Enschede, The Netherlands; \\ 2. HKU Theatre, HKU University of the Arts Utrecht, Utrecht, The Netherlands \\ j.p.weijdom@utwente.nl
}

\begin{abstract}
Collaborative Mixed Reality Environments (CMREs) enable designing Performative Mixed Reality Experiences (PMREs) to engage participants' physical bodies, mixed reality environments, and technologies utilized. However, the physical body is rarely purposefully incorporated throughout such design processes, leaving designers seated behind their desks, relying on their previous know-how and assumptions. In contrast, embodied design techniques from $\mathrm{HCI}$ and performing arts afford direct corporeal feedback to verify and adapt experiential aesthetics within the design process. This paper proposes a performative prototyping method, which combines bodystorming methods with Wizard of $\mathrm{Oz}$ techniques with a puppeteering approach, using inside-out somaesthetic- and outside-in dramaturgical perspectives. In addition, it suggests an interdisciplinary vocabulary to share and evaluate PMRE experiences during and after its design collaboration. This method is exemplified and investigated by comparing two case studies of PMRE design projects in higher-art education using the existing Social VR platform NEOS VR adapted as a CMRE.
\end{abstract}

\section{CCS CONCEPTS}

- Human-centered computing $\rightarrow$ Human computer interaction (HCI); Interaction paradigms; Mixed / augmented reality; Interaction design; Interaction design theory, concepts and paradigms; Collaborative and social computing; Collaborative and social computing design and evaluation methods; Ethnographic studies.

\section{KEYWORDS}

creative collaboration, embodied design, virtual reality, theatre, performance,

ACM Reference Format:

Joris Weijdom. 2022. Performative prototyping in collaborative mixed reality environments: an embodied design method for ideation and development in virtual reality.. In Sixteenth International Conference on Tangible, Embedded, and Embodied Interaction (TEI '22), February 13-16, 2022, Daejeon, Republic of Korea. ACM, New York, NY, USA, 13 pages. https://doi.org/10.1145/3490149. 3501316

\section{(c) (†)(2)}

This work is licensed under a Creative Commons

Attribution-NonCommercial-ShareAlike International 4.0 License.

TEI '22, February 13-16, 2022, Daejeon, Republic of Korea

(C) 2022 Copyright held by the owner/author(s).

ACM ISBN 978-1-4503-9147-4/22/02.

https://doi.org/10.1145/3490149.3501316

\section{INTRODUCTION}

The potential of using immersive head-mounted-display (HMD) based Virtual-Reality (VR) technology in design collaborations and training is steadily being recognized in fields like engineering design [48] and architecture [3]. Additionally, in a cultural context, VR technology is explored for its artistic potential by artists in interactive media, games, and performance. Consequently, offline and online festivals and conferences increasingly showcase a new hybrid type of artistic productions, which could be typified as what this paper calls Performative Mixed Reality Experiences (PMRE). Furthermore, the emergence of a new generation of so-called Social $V R$ platforms facilitates online social interaction and collaboration, which increases VR technology adoption for entertainment and work [30]. Similarly, working from home in co-located digital environments has become more common since the current global COVID-19 pandemic.

Kilteni and Slater et al. [28, 44] discusses how the human sensory apparatus responds to a virtual experience as real while cognitively knowing it is not. Other research shows that interactions in immersive VR connect to notions of embodied cognition, tacit knowledge, and learning $[10,24,41]$, especially when this embodiment is mediated through an avatar $[2,28]$. By purposefully including the physical body and space while using VR, a mix of physical and virtual spaces, objects, and participants become part of what this paper considers a Mixed Reality (MR) environment. In line with earlier artistic research on PMRE design [7], such an environment can facilitate embodied design strategies that holistically incorporate the sensory apparatus while cognitively reflecting on its embodied interactions. This enables, firstly, the generation of new ideas through embodied exploration and experimentation. Secondly, the development and testing of those ideas through embodied improvisational performance. And finally, the collective evaluation of its outcomes through a shared embodied experience. While Social VR platforms offer virtual environments for creative collaboration [21], little attention is given to purposefully incorporating the physical body and space as part of the collaborative design process. Hence, in this research project, an existing Social VR platform is selected, adapted, and implemented as a so-called Collaborative Mixed Reality Environment, or CMRE, enabling collective embodied design strategies in a mix of physical and virtual environments.

Using the body in an HCI design process context is, as Dourish suggests, an approach that "takes embodiment to be central to, even constitutive of, the whole phenomenon" [14:102], whereby interacting through the body as a meaning-making activity is inherently connected to its situated 'being-in-the-world' including its environmental and social context. Since the so-called 
'somatic turn' in Human-Computer Interaction (HCI) [32], embodied design strategies that originate from the performing arts have been developed for interaction- and engineering design purposes $[6,18,29,33,34,37,39,43]$. However, very few of them, both in the performing arts and HCI, have been implemented and studied in highly technologically mediated environments, like CMREs, especially in MR experience design processes. Considering these developments, the urgency to explore VR HMD-based CMREs for their creative potential through embodied design collaboration becomes evident, both in professional contexts as in art-, HCI, and engineering design education.

This paper proposes a method of performative prototyping using various embodied design techniques from the performing artand HCI design practices, including Wizard of Oz (WoZ) [11] techniques with a puppeteering approach. Additionally, it combines an integrated dual perspective of phenomenologically being in and dramaturgically looking at the designed experience. This performative prototyping method is the primary outcome of this study as a result of the execution and comparison of two consecutive case studies in higher art education with the goal to design PMREs through embodied design techniques using an adapted Social VR platform as a CMRE. This study questions how embodied design techniques can be incorporated in a CMRE for designing PMREs using a research through design [50] approach, whereby the design activities play a formative role in the generation of knowledge [46], and the researcher is involved in the experimental design activities [12]. As such, the design processes in the case studies are both the context of the study as well as examples of the execution of performative prototyping methodology. Consequently, in this paper, the resulting CMRE prototypes and aesthetic PMRE artifacts are considered secondary outcomes instrumental to and illustrations of the execution of this method. Therefore, these prototypes and artifacts will not be extensively described and analyzed in this paper.

This paper starts by outlining related work on Social VR as collaborative environments, existing embodied design methods in $\mathrm{HCI}$, and examples of PMREs, to contextualize this research and some of the key terms used. Then, the research method of this study is explained, followed by a project description of the two case studies. Next, the selection process leading to choosing the Social VR platform NEOS VR is briefly motivated. Subsequently, the design process of the two consecutive case studies, showcasing performative prototyping in practice, are described and compared through five distinct design activities: exploring, experimenting, performing, prespatializing, and evaluating. After which, the proposed performative prototyping method is described and summarized. Then, the implementation of different embodied design techniques and the proposed performative prototyping are discussed in more detail. Additionally, an interdisciplinary vocabulary is suggested to express, share, and evaluate the MR experiences in the collaborative design process and its outcomes. Finally, this paper concludes by discussing the implications of this study for MR design research specifically and its relevance for design collaborations in virtual environments in general.

\section{RELATED WORK}

Performative prototyping as a method for designing PMREs needs a CMRE and embodied design methodologies that combine concepts from $\mathrm{HCI}$ and the performing arts. In this chapter, I first briefly discuss related work on Collaborative Virtual Environments (CVEs) in relation to Social VR and its requirements for collective creative design processes. Second, I summarize key concepts on embodied design methods from $\mathrm{HCI}$ discourse in relation to concepts from the performing arts. Third, I describe examples of what I call Performative Mixed Reality Experiences (PMREs) to illustrate this emerging field. And finally, I briefly discuss research through-, and research in design as these approaches are used in this study.

\subsection{Collaborative Virtual Environments (CVEs) and Social VR}

Next to entertainment purposes, people increasingly use VR for collaboration in a shared virtual environment, often referred to as a Collaborative Virtual Environment (CVE). Benford defines a CVE as "virtual worlds shared by participants across a computer network" [5:79]. These open-world online virtual environments combine sandbox games and social computing components in what Messinger et al. metaphorically qualify as "a globally shared playground and workspace" [35:204]. In the last few years, the amount of VR-based training and collaboration tools has risen considerably. For example, a market overview of VR collaboration tools by the Institute for Immersive Learning shows more than ninety [21]. However, only eleven CVEs that afford shared meetings, training, and design collaborations are categorized as Social VR platforms. Furthermore, the Institute for the future considers that Social VR platforms should be "open-ended" [22:12], rather than a dedicated task-based application with a particular professional purpose. This 'open-endedness' makes Social VR platforms suitable for creative design collaborations and indicates a key requirement for a CMRE that facilitates embodied design techniques as part of its performative prototyping methodology.

\subsection{Embodied Design Methods in HCI}

Embodied design methods include the physical body and its direct experience in the design process, or as Wilde et al. formulate "enables all of a person's senses to be leveraged in an emergent design space" [49]. This forces the designer to not only mentally think through a design challenge but also physically move through its experience to come up with new insights and possible solutions. Designing, prototyping, and presenting through embodied engagement with the environment, objects, and other people, is an essential characteristic of theatre and performance practice, using improvisation techniques to devise new ideas and concepts and perform live experiences [16]. Also, in human-computer interaction (HCI), these techniques are used in ideation design phases through the concept of bodystorming. This general technique refers to "brainstorming activities that heavily rely on a rich bodily and situated engagement with the ideation process wherein designers use their bodies to enact design functionality or usage" [34:195]. The term has been used for many embodied design techniques like experience prototyping [6] and role-play [37].

However, often these techniques start from an existing applied design idea, artifact, and environment. This is why distinguishing whether bodystorming is used for idea generation, development, or testing, is helpful to determine the appropriate embodied design 
technique. For example, when bodystorming is aimed at idea generation, before a problem definition, and without a predetermined design artifact or context of use, Schleicher's embodied storming [39] or Márquez Segura's embodied sketching [33] offers a suitable focus. Embodied storming aims to shift from "an approach of defining user needs to a communicative mode of rapidly expressing ideas and proposals" by creating "stories or themes out of the things we observe around us" [39:49, 50]. Similarly, embodied sketching focuses "on initial explorative ideation phases as a way to open up the design space (v) at the experiential level" [33:6023]. An approach that is not unlike devised theatre, which begins its creative collaborative design process "without a script" [27].

Similarly, the idea of Wizard of $\mathrm{Oz}$ (WoZ) [11], a common technique in $\mathrm{HCI}$, resonates with the tradition of puppeteering in theatre through its concept of mediating one's performance through an object [26]. Traditionally WoZ in HCI can be used to create flexibility while user-testing, or as Dahlbäck et al. formulate, "the interaction is mediated by a human operator, the wizard, with the consequence that the subject can be given more freedom of expression" [11:259]. Which in context of a CMRE, as Dow et al. point out, also means that the design tools for the wizard interface should preferably be integrated directly into the design environment [15]. More recent developments and discourse offer helpful insights into applying these techniques in prototyping and presenting MR experiences $[7,20]$. For example, Lee combines bodystorming and WoZ techniques in MR environments through a method called Embodied Design Improvisation to "reveal and evaluate appropriate interactions for designers" [29:8]. However, as Kaplin points out, "while actors animate a sign vehicle from the inside out, using their own feelings, bodies, and voices, puppet performers must learn to inhabit the sign vehicle from the outside in" [26:29] This suggests that in the performative prototyping method proposed in this paper the use of WoZ with a puppeteering approach asks for an embodied awareness by the operator of its mediated performance of objects and events in the MR environment in response to other participants.

Through the concept of soma design, Höök explains that "the designer's (and end user's) lived body becomes a resource in the design process", whereby not only "movement-based engagements" but also "our subjective experiences, feelings, values, meaning-making", become part of the design process [18:16]. Additionally, NúñezPacheco advocates for reflection through inner-presence, an internal focus leading to insights that could otherwise be missed in actionbased methods with an external focus [38:7]. Soma design is based on phenomenology that tries to "study and describe reality as it appears in concrete experiences" [8:1228]. It builds on Schusterman's work on somaesthetics, a "critical study and meliorative cultivation of the soma as a site both of sensory appreciation (aesthesis) and creative self-fashioning" [42:111]. However, as Núñez-Pacheco points out, articulating and transferring this somatic knowledge is arguably difficult [38:4]. In this paper, as part of the performing prototyping method, I distinguish two ways the soma-based sensory appreciation can be articulated. First, as an inside-out phenomenological description of sensory phenomena (warm, small, dark), and second, as a somaesthetic evaluation towards a meaning-making interpretation (cozy, claustrophobic, scary).

\subsection{Performative Mixed Reality Experiences (PMRE)}

In this paper, I refer to performative mixed reality experiences (PMRE) when the body purposefully becomes part of the aesthetic appreciation of the MR experience itself, including seeing other participants and the technology itself perform within the context of the artistic concept. According to Dalsgaard [13], from an HCI perspective, the 'user' of a system is "simultaneously operator, performer, and spectator when interacting". However, the term performative is also used in this paper in the design and appreciation of the MR experience as a theatrical performance. Benford and Giannachi refer to mixed reality performances as "a term that is intended to express both the mixing of the real and virtual as well as their combination of live performance and interactivity" [4:1]. While classic theatre dramaturgy predominantly focuses on text and speech, concepts from post-dramatic theater consider that "breath, rhythm and the present actuality of the body's visceral presence take precedence over the logos" [31:145]. Additionally, it considers text, space, time, body, media, and technology as equal aspects in the meaning-making of the overall experience. Consequently, considering this "post-dramatic perspective", the used technology also becomes performative, offering an aesthetic performance within the artistic context of the designed MR experience.

\subsection{Research through-and research in design}

This paper focuses on embodied design techniques in CMREs, both researching through design activities as well as looking in(to) the process itself. As Dalsgaard specifies, research in and through design is typified by the "inquiry into the design process itself" through the iteration of a series of activities and artifacts, whereby the researcher's involvement is a "key catalyst for knowledge generation" [12:201]. As Zimmerman points out, "design research is framed as research on a condition that arises from a number of phenomena in combination, rather than the study of a single phenomenon in isolation" [50:496]. To research how embodied design techniques can be incorporated in a CMRE for designing PMREs, these three components had to be combined in the design activities while simultaneously developing them through iterative design cycles. In research through design, the design activities play a formative role in the generation of knowledge, whereby designers gain an "actionable understanding of a complex situation, framing and reframing it, and iteratively developing prototypes that address it" [46]. In this research, the development of a CMRE prototype is considered a required conditional environment in which the design process of PMREs through embodied design techniques can be studied. In this way, the CMRE prototype cannot be considered a separate outcome, addressing a complex situation or offering a solution to a research problem, without understanding the specific process of its use, summarized as the proposed performative prototyping method.

\section{METHOD}

This study questions how embodied design techniques can be incorporated in a CMRE for designing PMREs using a research through 
design [50] approach, whereby the design activities play a formative role in the generation of knowledge [46], and the researcher is involved in the experimental design activities [12]. Thus, in line with a research in- and through design [12] approach, it focuses on the design process of two consecutive case studies informing each other through an iterative cycle of design activities, generating and analyzing its process and outcomes.

First, a suitable Social VR platform was chosen to be adapted as CMRE for two case study projects in higher art education as described in the next chapter's project description. Then case study one (CS1) was conducted, after which the first analysis of its data informed the adaptations made for the execution of case study two (CS2), both in the CMRE prototype as in the design process activities. After finalizing CS2, a separate analysis of its data was made first. Then, comparing the two case studies focused on returning patterns and differences and the impact changes made by the teachers from CS1 to CS2. Finally, a summary of its key results has been compiled, proposing the performative prototyping method as its resulting outcome.

\subsection{Data collection}

For this study, activity-related data was collected by observing the students' design processes by my fellow teacher colleagues and me. Furthermore, after their course-related assessment, both student groups were interviewed through an online focus group discussion, moderated by me as the researcher. These conversations, design sessions in the adapted Social VR platform NEOS VR, and some physical theatre studio and classroom sessions have been screenand video recorded with the students' explicit prior consent. Additionally, informal evaluation conversations with the teachers have been held during and after the two case studies. I have recorded, transcribed, or summarized through field notes these conversations. In line with the research approach, I played an active role in the design activities, both as a teacher, 'client' and collaborator. Consequently, my research-based observations and notes have been purposefully kept out of the conversations with the student groups and teachers during the data collection process, enabling a later triangulation in the analysis phase comparing my findings with the observations made by the other teachers and students. Artifactbased data consist of the prototypes and final virtual environments in NEOS VR, including the entire process documentation by the students of CS1 permanently showcased in a Mixed Reality Expo in NEOS VR. Furthermore, the final PMREs have been documented with photos and video recordings, with the prior consent of the participants. Students have given additional consent after completing grading of their assignments, allowing for using the data for research analysis and publication, which has been approved by the ethics committee of the researcher's affiliated institute. While the final presentations of the PMREs are outcomes of the student's projects, they are considered one of the many design artifacts and will not be separately described as an outcome of this study.

\subsection{Data analysis}

The formative conversations and interviews with the students and teachers have been studied through a qualitative thematic analysis of the field notes and transcribed texts. A qualitative interaction analysis approach [25] has been taken to analyze the video- and screen-recorded design activities. This enabled the study of nonverbal behavior observed in the video recordings next to the participants' verbal communication. In analyzing the behavior, I primarily looked at the temporal structure of the recorded collective design process sequences using so-called "analytic foci" [25:57]. These were used to determine which design activity types would emerge as typical to embodied MR experience design processes and represent returning patterns in both case studies. Additionally, the Avatar-Based Collaboration framework [40] has been used to distinguish communicative-, navigation-, and object-related actions in the context of collaborations in virtual environments. This framework offers a semantic 'dramaturgical' approach, connecting who, when, where, and what with the goal-related actions and VR infrastructural elements. Marking these design activities enabled me to find emerging patterns in the design processes in both case studies whereby codes emerged out of this process of analysis rather than using a predetermined code-set. Also, this process of analysis of video - and screen-recorded activities has been iterated several times in dialogue with the findings coming out of the qualitative thematic analysis of the field notes and transcribed texts of the interviews. In this way, I compared my observations with those from the other teachers and students, verifying if others mentioned the patterns that I found or observations of others were found by me in the recorded video data. As mentioned, my observations as one of the teachers have not been shared with the teaching colleagues and students while conducting the interviews, enabling a later comparison after collecting all data. Additional debriefing interviews have been held with the teachers of both case studies to verify and possibly adjust the findings after the data analysis. These semi-structured interviews first asked open questions concerning the use of NEOS VR as CMRE, using embodied design techniques, and the design process in general, allowing the teachers to share their thoughts without knowing the findings of the research analysis. Then, the research findings were shared with the teachers to verify their accuracy, asking if anything needed to be adjusted, replaced, or added. As such, this paper offers the final findings and analysis by me after these debriefing interviews.

\section{CONTEXT OF PERFORMATIVE PROTOTYPING IN TWO CASE STUDIES}

This research incorporates two case studies to study embodied design techniques in CMREs and develop the proposed performative prototyping method iteratively. These case studies involve a courserelated design project in higher-art education executed by student groups with a similar assignment using the same pre-selected Social VR platform NEOS VR. Both case studies aim to design PMREs in CMREs through embodied design techniques while developing its method of designing. As such, this project is not offered to the students as a course to teach PMRE design through performative prototyping in a CMRE. Instead, this project was offered as an iterative process of aesthetic embodied experiential experiments to explore the artistic potential of mixed reality technologies and to share its various outcomes with peers as a result.

The first case study (CS1) involves a twenty-one-week project, starting at the beginning of September 2020 at the department of 


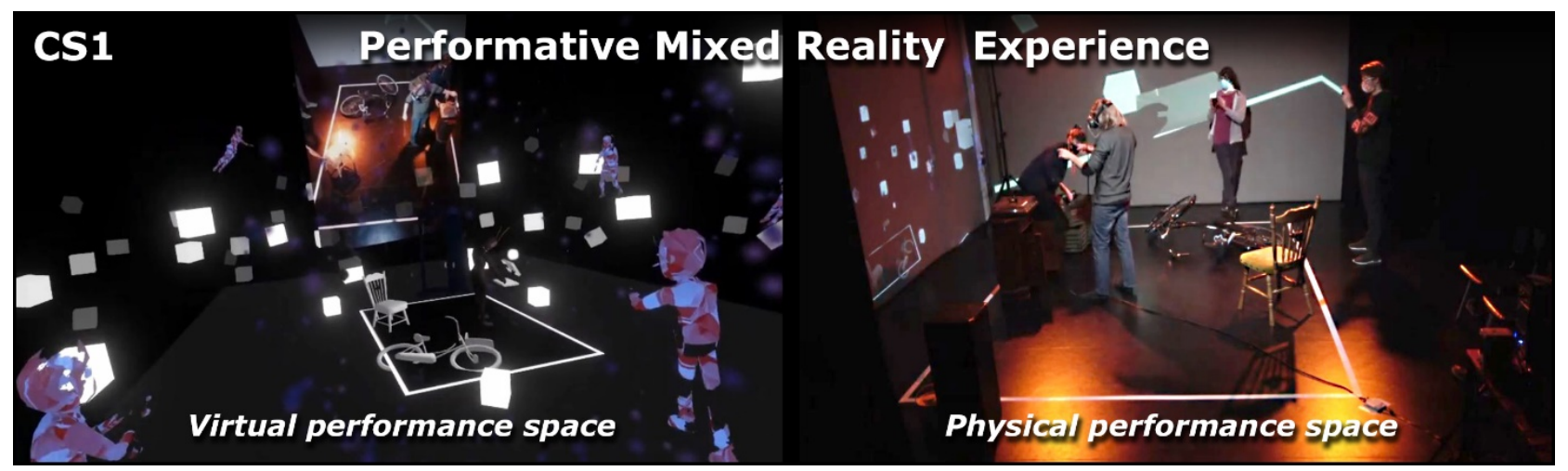

Figure 1: final presentation of PMRE from case study 1 student group combining a virtual and physical performance space.

Games \& Interaction (G\&I). The team consisted of six bachelor of art (BA) students in their third and fourth year at a University of Arts, focusing on various disciplines (game-art, -development, -design, and interactive performance). And additionally, one thirdyear bachelor of science (BSc) Game Technology student from another University. This project was coached by three teachers, two from the G\&I department and me as a teacher from the Theatre department. In addition, I also functioned in this project in the role of 'assignment giver' or 'client' of the student team.

At the start of the project, the students were given the following assignment: Create a meaningful and embodied mixed reality experience using NEOS VR and (several) physical locations for (minimal) 5 participants. This project showcases explorations in new ways of immersive storytelling, multiple levels of user participation, and innovative use of mixed reality technologies. The assignment explicitly required to use embodied design techniques to explore, develop, and present intermediate and final outcomes. CS1 connected to the Metamovie project Alien Rescue by Jason Moore [36] as an inspirational example of immersive storytelling in NEOS VR with live performers and participants. Furthermore, the assignment required an online exhibition in NEOS VR that would show the research, design process, and analysis by the students of the PMRE.

The second case study (CS2) involves a ten-week project, starting at the beginning of February 2021 at the Department of Theatre of the same Art University. The team is comprised of seven secondyear BA students studying Interactive Performance Design (IPD). The assignment for this project is mostly identical to that of the CS1, except that it didn't require an online exhibition and provided an external artistic context by connecting to the existing professional project Enter New Babylon [9]. This project translates the utopian future vision of the Dutch artist Constant from the 1970s into a contemporary MR experience to be showcased two months after the end of the students' project. As such, the IPD students were asked to offer an inspirational preliminary design of a PMRE within this external context offering their contemporary utopian, or dystopian, future visions.

The students were encouraged to employ a high degree of quickprototyping in both projects through iterative design cycles. Both student teams had no prior know-how in using Social VR as a collaborative design and presentation platform for designing PMREs. Consequently, in each project's timeline, everybody needed to get in VR, learn the Social VR platform's workflow, prototype various ideas, get expert input and training, design the experience, and finally, test and present its results to local and remote participants. The presentation of the PMRE of CS1 [17], see Figure 1, took place in December 2020, CS2 presented their PMRE [9] in April 2021.

\section{THE DESIGN PROCESSES OF TWO PMRE PROJECTS}

This chapter compares the PMRE design processes of the two case studies, resulting in the proposed performative prototyping methodology. First, the two consecutive case studies are compared through five design activity types, highlighting the key findings concerning using embodied design techniques in a CMRE creating PMREs, followed by the description and summary of the resulting performative prototyping method. These results are then discussed in the following chapter. However, to study collaborative embodied design processes of a PMRE, a CMRE prototype that can facilitate such techniques had to be realized first.

\subsection{Using a Social VR platform as CMRE suitable for embodied design techniques}

To efficiently use limited time and resources, I decided to choose and adapt an existing Social VR platform as the basis for a CMRE rather than developing an entire system from scratch. As such, for this research, the right Social VR platform had to be selected first based on its potential for creative collaboration, facilitation of embodied design techniques, and possibilities to connect to physical environments, objects, and people. Using and adapting a suitable Social VR platform allowed for utilizing its built-in networked, multi-user, avatar-based, VR-ready, real-time 3D computer-generated virtual environment, whereby for the case studies only connections to the physical environment had to be implemented within the already existing system. Out of several Social VR platforms that held the potential for creative collaboration, NEOS VR [45] was selected for one important reason; it provides a workflow where most creative design steps could be performed in real-time inside the VR platform itself. Therefore, the capability to do the designing, coding, 
prototyping, and sharing from within its VR environment allows for a continuous flow of embodied design strategies and experiential learning. Thus, potentially stimulating exploration, experimentation, performance, and evaluation within the interdisciplinary group based on a shared embodied collaborative experience.

\subsection{Examples of performative prototyping in two consecutive case studies compared through five design activities}

This paper's proposed performative prototyping method emerged from the comparative analysis of the two consecutive case studies with a research in and through design approach. In this section, the design processes of the two case studies are compared through five distinct collaborative embodied design activity types, namely exploring, experimenting, prespatializing, performing, and evaluating. I identified these five activity types by analyzing the design process, artifacts, and interviews with the CS1 and CS2 participants as distinctive returning patterns in both case studies. As such, these five activity types are used in this paper to illustrate and exemplify the proposed performative prototyping method by focusing on the specific characteristics of the embodied design process of a PMRE and its facilitation by the adapted Social VR platform NEOS VR as a CMRE.

5.2.1 Exploring. To enhance the students' know-how in using a Social VR platform, they were assigned to explore the workflow and tools related to NEOS VR as CMRE. Additionally, both student groups explored the design of PMRE's by looking at other artists' work. As such, exploring is used in this paper as the direct embodied playful engagement with a MR environment.

The teacher team organized so-called 'virtual field trips' in NEOS VR to expose the students to virtual worlds made by the platform's online community. First, students were asked to experience these environments from an inside-out perspective by focusing on their somatic sensations. Then, students were asked to articulate meaning based on the experiential effect of the environmental spatial and atmospheric qualities, followed by distilling the key design components that induced these experiences. Thus moving from a phenomenological description to a somaesthetic evaluation. Additionally, lectures were offered from an outside-in perspective by showcasing VR art installations, immersive theatre works, and PMREs, discussing their meaning through concepts from theatre dramaturgy. Finally, the artistic context was introduced by meeting the professionals that were involved in the external projects. In CS1, this included an exclusive preview of the actual Alien Rescue experience, with the opportunity to talk to the director, design team, and actors afterward, practicing both perspectives in the same project. In CS2, an elaborate lecture and Q\&A on Constant's work was organized with the director of the Constant Foundation, initiator of the Enter New Babylon project.

Analysis of both projects shows that the students of CS1 had more difficulty than those of CS2 to observe, recognize and describe phenomenological sensations responding to the virtual environments. For example, when exploring NEOS VR worlds, CS1 students would comment on shading and level-design aspects of the virtual space but could not find words when asked how the environment makes them feel. Instead, they would speak in terms of personal aesthetic preference by pointing out what they liked or not liked. However, both groups had to practice the somaesthetic and dramaturgical evaluation of the environments, whereby the CS2 students grasped the concept of performativity faster due to their theatre-based background.

To encourage exploring the workflow and design tools of NEOS $\mathrm{VR}$, the students in both groups were given assignments to create personal MR environments individually. While CS1 mostly did these explorations in VR, partly due to COVID-19 restrictions, the students in CS2 were actively encouraged to begin by exploring physical spaces and translating these into physical scale models, adding digital light, textures, and (moving) images with projection mapping techniques. After these physical explorations, they were asked to translate their ideas into a virtual environment, which was first done in Mozilla Hubs in so-called desktop mode due to the late availability of VR hardware. Parallel to these explorations, the CS2 students received structured technical training in NEOS VR, including introducing the in-VR visual programming system called LogiX.

Both groups of students acknowledge that learning the workflow and tools of NEOS VR takes significant time and effort. This time was given to the students of CS1 without structured training, which seemingly undirected time-span affected the group's sense of purpose or design focus. Consequently, a more structured approach in CS2 was employed by offering a predetermined artistic context and technical instruction sessions in NEOS VR, also considering that the duration of CS2 was only half of the time of CS1. This structured approach was acknowledged by the CS2 students as very helpful, both artistically and technically.

5.2.2 Experimenting. After exploring artistic and technical possibilities, emerging design challenges were translated into initial ideas for several MR environment prototypes. In this paper, experimenting is used as a design activity type characterized by trying out creative ideas by designing a MR environment that is explored, adapted, and developed by direct embodied engagement of the designers and other participants. In this process, an embodied understanding of the idea and its design challenges offers insights to direct the experience or opens up new ideas and challenges evaluated through their aesthetic and dramaturgical potential.

The CS1 students primarily based their ideas on the Alien Rescue project with its inherent design challenges shared by its director Jason Moore relating to new ways of immersive storytelling and multiple levels of user participation. These challenges were translated into creative concepts for six prototype worlds in NEOS VR, which were developed by trying them out with peers, teachers, and members of the platform's community. For example, students experimented with assigning 'roles' to participants as extras, fixating their position in the virtual environment, or with new narratives by showing changing 'still-life scenes' by allowing the participant to switch the light on and off. Additionally, as the left photo of Figure 2 shows, the students experimented in their relatively small physical project space with tactile MR objects by connecting trackers to physical objects, like a desk chair, translating its location data to the virtual space. Additionally, they experimented with multiple audience perspectives by attaching a tracker to a mobile phone, 


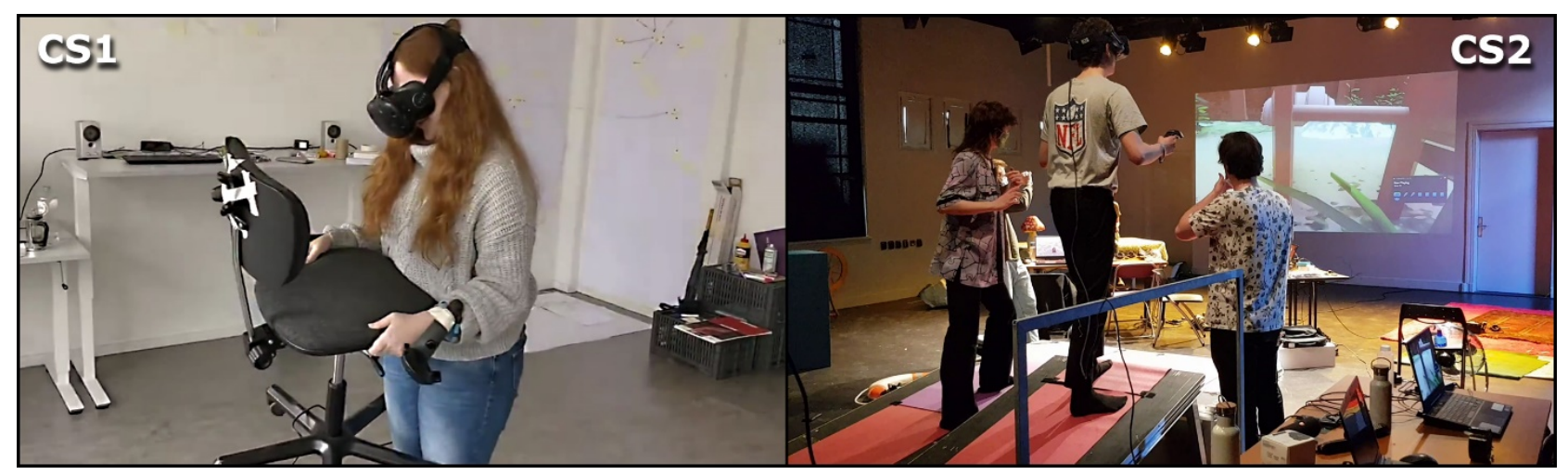

Figure 2: Left: CS1 Experimenting with desk chair as MR object. Right: CS2 experimenting with MR space with slanted floors.

offering a real-time view into the overlapping VR space, effectively creating a tangible MR camera.

The CS2 students were offered a theatre studio for a week halfway through the project to experiment with New Babyloninspired NEOS VR environments in relation to the physical space. Physical environments were constructed to experiment with design challenges relating to physical perception and locomotion in MR spaces. For example, as the right photo of Figure 2 shows, one physical setup consisted of several slanted surfaces in irregular angles on which a participant wearing a VR HMD had to negotiate a suggested route. Inside VR, the participant saw a surreal virtual environment with architectural elements placed at irregular angles, thus doubling a disorienting effect concerning determining a level horizon and maintaining balance. Another setup experimented with the sense of touch through connecting physical textures, like a rug, plants, and a wind machine, with virtual objects and environments. As with CS1, these CS2 experiments were tested on peers and teachers, using their feedback to adapt the setups through several iterations.

These design activities were aimed at generating and developing ideas by physically experimenting with what was created. While the CS1 students produced several rich virtual environments, they struggled to find meaningful relations to physical space and objects to develop their ideas further in their self-organized sessions. Additionally, they focused predominantly on whether the participants understood what to do rather than how they felt or what the environment caused them to want to do. In comparison, the CS2 students benefitted from working in a theatre studio experimenting with MR objects and environments starting from the physical space. The teachers coached them to experiment with what they had created, evaluated the resulting experiences, and determined the next design iteration. Offering a clear artistic theme in CS2 unrelated to the inherent complexities of MR experience design encouraged the students to start exploring and experimenting with creative ideas by making work themselves.

5.2.3 Prespatializing. Prespatialzing is a new term used in this paper to refer to a design activity that employs an embodied approach to previsualization techniques during the design process. It combines two experiential perspectives of looking at and being in a 3D virtual scale model of an intended physical setup while evaluating and making spatial adjustments in real-time.
Having experienced and iterated several prototypes through experimenting, the students developed their ideas into more robust artistic MR experiential concepts. Both groups were offered to build and test their concepts in a theatre studio before the final presentation. Virtual scale models of the actual presentation venue were constructed in NEOS VR to determine how the final setup would function before working at the actual location. As Figure 3 shows, these virtual scale models allowed the student-teams to previsualize the final setup from an outside-in perspective and enabled them to experience the space from the inside-out relating to the environment in a more embodied way. This dual perspective capability is due to the native affordance of Social VR to scale oneself in relation to the virtual environment. This self-scaling affords the designer to enter the 'scale model' as a 1:1 virtual environment, while others are still observing you from the 'outside' while being in VR, possibly in a different scale relation.

The team of CS1 used this design activity type to experience, test, and evaluate complex multiple user perspectives in relation to the MR space. The CS2 students used the virtual scale model to determine the positioning of the three inter-related works and how participants could be directed spatially from one to the other. These virtual environments were used to present the final concept to the teachers, try out multiple user perspectives to understand the dramaturgy of the experience from different angles, and solve many logistic problems relating to the physical space and technical infrastructure.

Utilizing virtual scale models offered both students teams an opportunity to fine-tune their concepts before entering the final venue in the last stage of the project. This embodied experiential type of previsualization resulted in artistic improvements and a logistic preparation that otherwise would have taken time in the actual physical space. This design activity type thus freed up precious moments to develop and rehearse the performative aspects of the MR experience. However, it also became clear that this collective switching between the different artistic, dramaturgical and logistical perspectives within the same session needs practice and guidance.

5.2.4 Performing. In this paper, performing is used as a design activity that considers and utilizes all participants, the MR environment, and the used technology as performative elements in the 


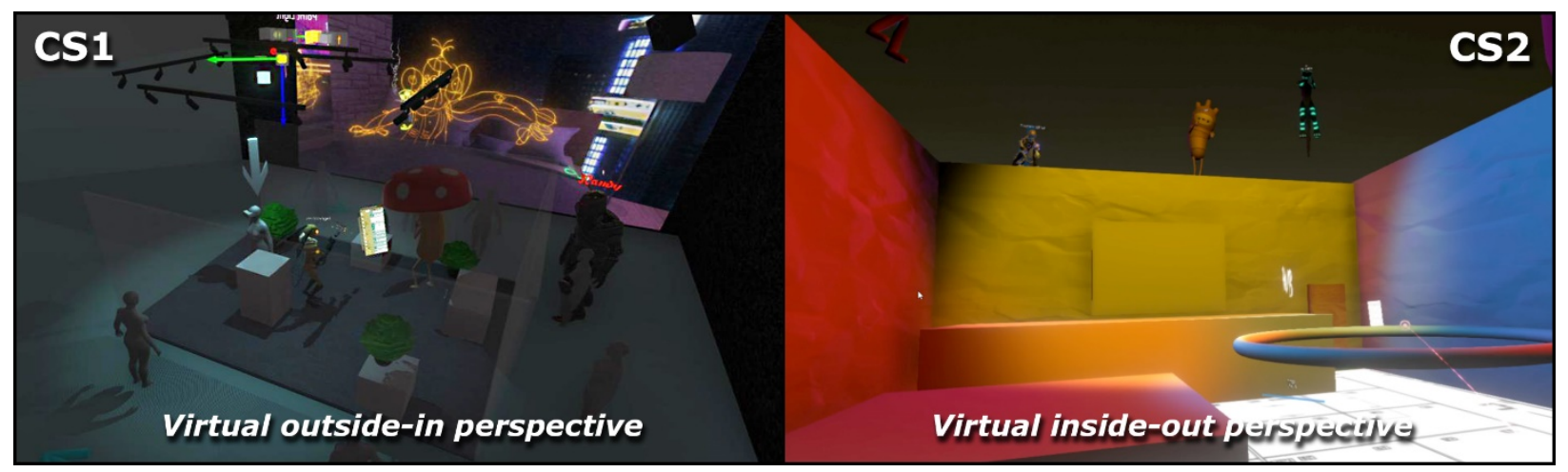

Figure 3: Left: CS1 testing user perspectives. Right: CS2 placing the three works in the virtual scale model of the physical theatre space.

aesthetic appreciation of the design process and its outcomes. Additionally, PMREs are live and often involve an audience that engages as co-performers with the experience through different levels of participation. Therefore, the students were encouraged to incorporate various performative strategies to welcome, instruct and direct the participants through the experience. This interaction with the participants could be done directly as performers or indirectly as puppeteers through the live operation of technical 'controls', triggering medial events in the MR environments in real-time. Both groups decided to use both strategies in their final presentations. Consequently, they had to learn, try out and rehearse live performative acts, which needed to be partly improvised in response to relatively unpredictable participants.

The students of CS1, primarily trained in the field of game design, had no prior experience with live performative improvised interactions with participants within a theatrical frame. However, for their MR experience, they could not just explain the rules of the interaction to the participants but had to learn how to become part of the theatrical experience by turning into performers. Some of the students of CS1explicitly expressed a degree of discomfort knowing they had to do so. Nonetheless, while the students of CS2 did receive theatre training, they also needed to learn how to perform in response to live participants. For this reason, several live improvisation sessions were offered in both projects to learn how to respond naturally to participants while directing the experience, either directly as performers or indirectly as puppeteers. In CS1, one of these improvisation sessions was held in NEOS VR, enabling exclusive training by an external international expert in directing participants in PMREs.

As Figure 4 shows, the physical media cues and transitions of theatre lighting and sound were live operated by the student-teams in the physical studio spaces. Simultaneously, LogiX-based virtual sliders and buttons were used in NEOS VR as WoZ controls to trigger virtual light, sound, locomotion schemes, and world transitions. Mapping those virtual controls to a physical Midi-pad interface through WebSockets and OSC created a direct connection with the physical environment. Furthermore, these physical controls enabled a corporal inside-out sensitivity of the operators to the timing of outside-in mediated responses to physical participants. As such, these operators functioned as puppeteers, performing the MR space in interaction with the participants and other performers.

5.2.5 Evaluating. In the context of art-based projects, every creative design activity needs moments of aesthetic evaluation to recognize valuable findings and determine the following step. However, during embodied design activities, this alternation between doing and thinking can happen rapidly in quick-iterations making a clear separation challenging to distinguish. Thus, in all the design activities, a certain degree of evaluating is continuously present but differs in its focus depending on the type of activity, the ideas and challenges relevant to that moment, and the overall project's design phase.

Analysis of the two projects shows that a vocabulary to express and evaluate the individual and collective experience to collaboratively design MR experiences through embodied design techniques is needed. Both student groups struggled in varying degrees to express phenomenological descriptions and somaesthetic evaluations in their direct embodied response to the MR environments. The articulation of how a MR environment feels through physical sensations needed to be exercised throughout the different design activities. Seemingly simple observations like the size of the space, the color temperature of the lighting, the resonance of the soundscape needed sometimes to be pointed out before they became part of the conscious and collective design evaluation. Also, they lacked a vocabulary and concepts to evaluate the artistic and dramaturgical implications when observing themselves or others perform in these theatrical spaces. Additionally, making a connection to how the body was responding from the inside-out, using a phenomenological and somaesthetic perspective, to how the experience would work from the outside-in, using a dramaturgical perspective, proved to be quite a substantial learning curve. While the theatre-based students also needed to practice this skill, especially non-theatre students, teachers, and external professionals lacked a vocabulary and concepts to express, share and evaluate their MR experiences using these perspectives.

\subsection{Performative prototyping}

In this study, a particular collection of embodied design techniques and protocol for its use, with multiple inside-out and outside-in perspectives, combined with an interdisciplinary vocabulary to express 


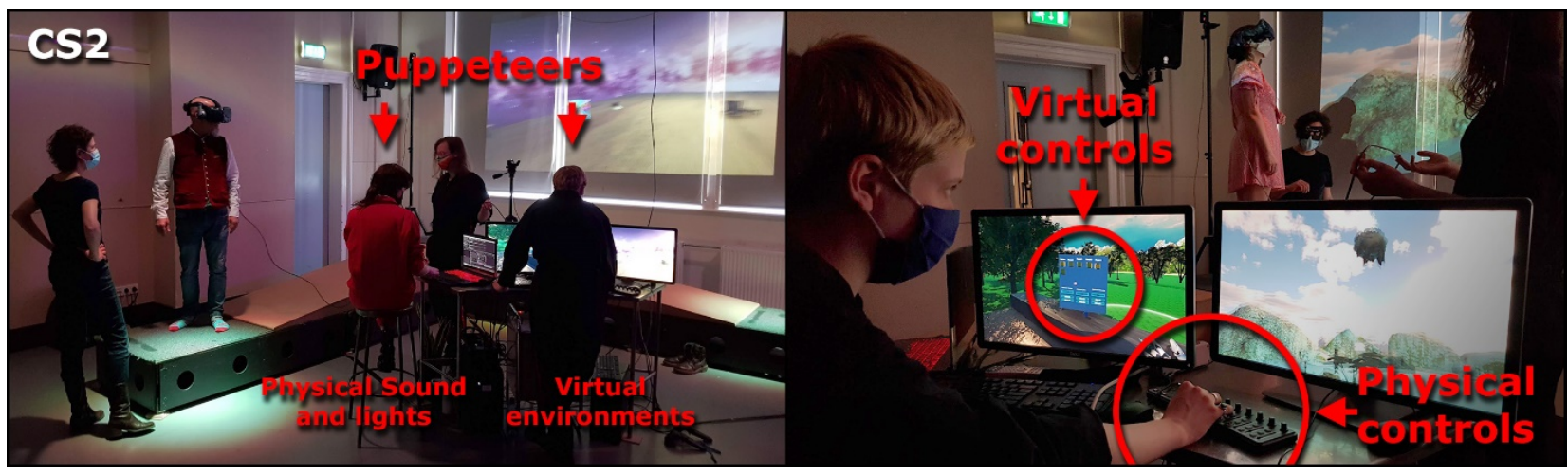

Figure 4: CS2 puppeteering physical sound, lights, and the virtual environment with physical and virtual controls.

and evaluate the individual and collective experience, emerged from comparing the two described case studies of this research. I call this a performative prototyping methodology.

Performative prototyping employs several embodied design techniques, summarized as bodystorming [34], combined with WoZ strategies $[11,15,29]$ with a puppeteering approach [26], foregrounding the performative focus of this method. Deciding which bodystorming techniques to use depends if the design activity is aimed at idea generation, development, or testing. Firstly, idea generation is facilitated through embodied storming [39] and embodied sketching [33]. Secondly, experience prototyping [6] or Embodied Design Improvisation incorporating WoZ techniques in MR [29] are suitable for development. And thirdly, testing can be done through Role-play [37]. However, these techniques overlap, giving different results depending on the degree of improvisation used to allow for ideas to emerge and develop or if a prewritten script is followed to test these ideas. Additionally, each embodied design technique is approached by combining an inside-out inner-presence perspective, focusing on internal corporeal sensations in response to the MR experience, with an outside-in movement-based perspective, focusing on external physical (inter-) actions.

Performative prototyping incorporates an embodied phenomenological- and somaesthetic inside-out perspective with a dramaturgical outside-in perspective to identify and interpret the meaning of the MR experience, whereby one's own body both performs its responses to, and becomes performative in, the MR environment. As such, it incorporates Dalsgaard's performing perception [13], where a user is engaged and aware of the act of interacting, perceiving, and performing, whereby the 'user' is considered the designer of the MR experience. Additionally, performative prototyping uses an interdisciplinary vocabulary to express, share, and evaluate the aesthetic qualities of these activities' experiences and creative outcomes to further the design process. The vocabulary of performative prototyping combines phenomenological description and somaesthetic evaluation terminology with a simplified artistic dramaturgical understanding of performativity from post-dramatic theatre practice and mediaand performance studies. Additionally, it connects to specialized language relating to MR technologies in ways that 'solutions' to design 'problems' are not dominated by purely artistic or technical considerations but rather emerge from their dialogue.
Performative prototyping uses exploring as a design activity type to try existing MR experiences and systems through direct embodied playful engagement. These explorations preferably are collaborative, playing with tools and affordances of existing systems or doing 'virtual field trips' to artistic MR experiences using the inside-out and outside-in perspectives. This activity type is essential to understand the potential of the emerging field of mixed reality experience design, both artistically and technically, as well as provide an opportunity to familiarize oneself with and train the dual perspectives and interdisciplinary vocabulary of performative prototyping. Experimenting as a performative prototyping design activity type is characterized by trying out creative ideas by designing a MR environment that is explored, adapted, and developed by direct embodied engagement by the designers or other participants. Keeping the responses of the MR environment flexible using WoZ techniques with a puppeteering approach, spontaneous design iterations can be performed through live embodied improvisations. As such, performing can be used as a generative design activity as part of experimenting. However, in this paper, performing is also highlighted as an activity type that engages other participants in a more elaborate MR environment to test and fine-tune its experience. In this case, the designer possibly performs within and as part of the MR experience through direct or mediated interaction. Furthermore, performative prototyping suggests prespatializing as a distinct design activity type that utilizes the inherent affordance of NEOS VR to scale oneself in relation to a virtual environment. This effortless scaling affordance enables the collective sharing of looking at or being in a digital scale model while making adaptations, preparing the physical setup in the intended location for the MR experience before being allowed access to the actual venue. Finally, while evaluating is mentioned as a separate design activity type, it permeates throughout all others. However, in the context of performative prototyping shifting continuously from direct embodied experience to communication and evaluation of its quality and potential meaning, incorporating both inside-out and outside-in perspectives, evaluation needs to be recognized as a distinct mode of operation by the designer and the team. 


\section{DISCUSSION}

So far in this paper, a description of the performative prototyping method has been given, combining specific embodied design techniques in a CMRE with a dual inside-out and outside-in perspective approach. This method emerged from comparing the two consecutive case studies, aiming to design PRMEs through embodied design techniques within the adapted Social VR platform NEOS VR. In this chapter, I discuss the implications of these findings in three parts. First, I will look at the implementation of different embodied design techniques within these technological environments and how their application can vary depending on the design activity and phase of the project. Then, I will discuss challenges relating to using the performative prototyping method for the ideation, development, and evaluation of PMREs. Finally, I will discuss the broader implications of these findings and the usefulness of the performative prototyping method for MR design research.

\subsection{Implementing embodied design techniques in MR experience design}

This study questions how embodied design techniques can be incorporated in a CMRE for designing PMREs. The embodied design techniques can be used with a different focus in different phases of the design process in various sequences within short design iterations to generate, develop, or test ideas. For example, bodystorming as an idea-generating activity moves away from pre-scripted, usercentered, and product-related design processes. Instead, the activity should allow a more responsive, collective, and free-flow bodystorm type to take place in what Schleicher calls embodied storming [39] or Márquez Segura embodied sketching [33]. In both case studies, these techniques were especially stimulated in the experimenting and prespatializing design activities, where new ideas, forms, and concepts could still emerge out of the process. Nevertheless, both these approaches to bodystorming are action-, or movement-based $[1,34]$.

Especially within the artistic context of this project, the aesthetic appreciation of a MR experience is not necessarily based on (physical) actions or the necessity to constantly physically move. Consequently, a different approach to embodied design was needed to allow for states of being instead of doing in the design activities. Soma design focuses on becoming sensitive to experientialcorporeal states that are not only action- or movement-based. Especially Núñez-Pacheco's emphasis on reflection through innerpresence [38] was particularly relevant for sensitizing the students to their bodily responses to the MR environments. This sensitivity was already essential in exploring other artists' MR works by first going through the experience, then watching, reading, or talking about the experience. In the bodystorm sessions of the students, visual, auditive, kinesthetic, proprioceptive, and vestibular sensations were recognized and discussed as valuable input for design considerations, as well as more internalized interoceptive sensations identified through a degree of inner-presence. However, as mentioned in the introduction, Kilteni and Slater et al. [28, 44] point at the dichotomy between real embodied sensations in response to the virtual environment while cognitive knowing it is not real. This dichotomy posed an artistic challenge for the students in their design explorations and experiments, trying to express, share and evaluate their experiences dramaturgically. Understanding this experiential contradiction did not need to be 'solved' to enable a 'perfect illusion' in the MR experience was a new concept to the students. Instead, embracing the dichotomy of MR experience as an artistic opportunity through its potential dramaturgical tension of colliding experiential spaces often proved too complex in their design considerations.

While sensitivity to bodily sensations remained essential throughout the different design activities as a source of experiencebased design-related information, the students of CS1 tended to mostly use embodied design strategies for testing ideas also when new ideas needed to be generated. Additionally, they expressed discomfort in needing to use improvisational role-play techniques to develop performative interactions with participants. Both could be explained by their disciplinary background being rooted in game design and development, implying that more know-how from theatre and performance was needed within the team. However, the theatre-based students of CS2 also needed guidance and support in implementing these techniques in their design process. Whereby an increase of this support resulted in a considerable acceleration of design iterations leading towards meaningful MR experiments in only half of the project time compared to CS1. This structural support aligns with Johnson-Glenberg's nine design principles for embodied learning in VR [23], especially the guidelines to Scaffold cognitive effort, provide guided exploration, and co-design with teachers. Additionally, the study shows students felt more comfortable starting with and within a physical environment that they already know, rather than getting lost in an endless virtual space needing to find their way back. This beginning with the physical might be related to ideas of embodied cognition, considering physical interaction with the environment as the basis for learning and understanding the world [41].

These findings suggest that many of the struggles, confusion, and discomfort that both student groups demonstrated in implementing embodied design techniques and integrating the inherent experiential paradoxes concerning MR environments are related to their inexperience as designers. Thus the different forms of bodystorming need to be practiced as well as evaluating them dramaturgically. Also, becoming sensitive to corporeal sensations and translating them into design-related information and decisions must be learned by training [47]. While this inexperience is undeniably a factor in their design process, this doesn't mean that professionals would not find this challenging in their design practice. Additionally, while both professionals from the performing arts and HCI use embodied design techniques, they are not necessarily experts in applying these techniques productively within the interdisciplinary field of PMRE design.

\subsection{Challenges of performative prototyping in practice: training interdisciplinary skills and vocabulary}

This paper focuses on how specific embodied design techniques within CMREs can be translated into a method for ideation, development, and evaluation of PMREs for professionals and students in art-, engineering, and HCI design practice. It proposes a performative prototyping methodology combining specific embodied 
design techniques in a CMRE with a dual inside-out and outside-in perspective approach. This method emerged from comparing the two consecutive case studies, which illustrate in practice how performative prototyping can be applied in design processes of MR experiences using a CMRE. Furthermore, the case studies exemplify how performative prototyping methodology in CMREs allows for fast iterations of quick prototyping design cycles, incorporating collective full-body engagement and direct generation, adaptation, and evaluation of its MR experience. These iterations result in the extensive exploration and development of ideas, meaningful MR experiences, and possibly new applications of MR experience design.

However, as discussed in the former part, the findings also show that applying embodied design techniques within the interdisciplinary field of MR experience design is not easy and needs to be trained, both by students and professionals. Moreover, being sensitive to bodily sensations in a MR environment, simultaneously interpreting their design implications, adds to the complexity of skillful execution of the performative prototyping method. Furthermore, the designer is also asked in this method to combine these phenomenological-and somaesthetic inside-out perspectives with an outside-in perspective, qualifying performative actions and evaluating its meaning through a dramaturgical lens. As such, the designer is asked to inform design decisions by rapidly alternating between being the participant and the designer of the experience.

While the earlier mentioned lack of professional experience of the students and their limited disciplinary know-how affected the execution of these dual perspectives in the collaborative design process, it is arguably also a challenge for design professionals. From the debriefing interviews with the teacher experts and my own professional expertise, I can say that these multiple perspectives cannot be held simultaneously in our awareness within the experience of a MR environment while designing. Instead, developing professional skills in using the performative prototyping method is about knowing what, when, and why to shift in embodied design technique and perspective in relation to what the collective design process needs in that instant.

Additionally, to express, share, and evaluate these perspectives collectively with and within the design team while being in the MR experience requires the training of an interdisciplinary vocabulary that all participants understand. As Núñez-Pacheco indicates, articulating phenomenological descriptions and somaesthetic evaluations is difficult [38:4], and translating these into design considerations needs to be trained by repetition [47]. Furthermore, existing concepts from theatre dramaturgy need to be reconsidered through a post-dramatic perspective [31], incorporating both the aesthetic and instrumental performance of MR technology as an inextricable part of the MR experience design process and its outcome.

Finally, due to the interdisciplinary workflow and vocabulary used in PMRE design, designers must be willing to re-invent and adapt their theoretical and practical know-how and learn from other disciplinary fields. Therefore, the design activity of exploring is suggested as an intricate part of the performative prototyping method in designing MR experiences. Exploring enables ongoing discovery of the emerging field of MR experience design, as well as training the dual perspectives by collectively sharing and evaluating existing artistic PMRE works. Furthermore, while not discussed in this paper extensively, the analysis of the case studies shows that the students needed quite some time to learn the interface and tools of NEOS VR as adapted CMRE. This suggests that with engaging in the new field of MR experience design, new computer-aided design tools suited for embodied design techniques also need to be learned. However, as Hornecker points out, learning these tools can be a 'mindful activity' whereby we "are most happy when we feel we perform an activity skillfully and gracefully even if it took us a painfully long time to get to this point" [19:23].

\subsection{Broader application for performative prototyping in MR design research and practice}

In this project, a specific Social VR platform has been selected and adapted to a CMRE configuration, suggesting that others can easily employ, develop, and study embodied design techniques in MR environments. However, implementing a performative prototyping methodology for designing PMREs in higher art- and engineering design education in such a CMRE might seem rather specific and possibly relatively narrow in its range of applications. I nonetheless suggest that its praxis provides a creative design method and environment relevant to the broader field of Collaborative Virtual Environment (CVE) supported HCI design practice. Namely, performative prototyping offers a method to facilitate interdisciplinary collaborations that purposefully re-incorporate the physical body and live experience at the center of the technologically mediated design process. It can be used to quickly prototype experiential and spatial concepts and is suitable for designing VR or MR applications, including Augmented Reality (AR). It provides a real-time embodied method for generating, adapting, and evaluating design ideas and concepts, focusing on the embodied experience of environments, objects, and fellow participants. Furthermore, it offers multiple inside-out and outside-in perspectives that provide a comprehensive view of the whole experience, potentially integrating corporal and cognitive processes as well as the individual's experience in relation to its social context. This multi-perspective method provides the design team with a rich approach to its creative process and hopefully stimulates more exploring, experimenting, performing, prespatializing, and evaluating of meaningful products and experiences.

\section{CONCLUSIONS AND FUTURE WORK}

The outcome of this study proposes a performative prototyping method combining a collection of embodied design strategies with dual perspectives and interdisciplinary vocabulary to facilitate creative design collaborations in Mixed Reality (MR) environments. It presents how a selection of embodied design techniques summarized as bodystorming can be combined with WoZ strategies using a puppeteering approach, enabling embodied design improvisations. The specific application of the different bodystorming techniques depends on whether the design process is focused on generating, developing, or testing ideas and concepts. Additionally, this study motivates the conditions for adapting and utilizing a Social VR platform as a Collaborative Mixed Reality Environment (CMRE) for creative art- and engineering design processes to use the performative prototyping method for the design of MR experiences. Using 
and adapting the Social VR platform NEOS VR as a CMRE, enabled student design teams to employ fast design iterations interacting with physical and virtual objects, people, and environments.

In performative prototyping an embodied sensitivity to inner and outer sensations as well as the perception that all elements become inherently performative in quick-prototyping sessions allows for an alternating dual perspective between phenomenologically being in and dramaturgically looking at the designed experience. These inside-out and outside-in perspectives need an interdisciplinary vocabulary that combines phenomenological description and somaesthetic evaluation terminology with a simplified artistic dramaturgical understanding of performativity from post-dramatic theatre and media and performance studies. Also, this study illustrates through the proposed performative prototyping design activity type of prespatializing how virtual scale models can be used to present, prepare and test the final MR setup in the context of its intended physical presentation location.

However, using a Social VR platform as a CMRE for collective creative design processes of MR experiences necessitates adapting learned disciplinary workflows. Also, the proposed selection of embodied design strategies needs further elaboration to be structurally applied in art- and engineering design practice and education. Furthermore, technical configurations to adopt a Social VR platform to a CMRE or embed WoZ controls in a MR system could be specified more clearly. And finally, developing an interdisciplinary vocabulary combining concepts and know-how from the performing arts, $\mathrm{HCI}$, and engineering design is an ongoing study. While some of this information is embedded as initial examples in the prototypes and processes of the case studies, it would take too much space to describe them all in this paper.

Still, this paper contributes to this ongoing work by offering the performative prototyping method as a first step in combining embodied design techniques with a dual perspective and suggestions for an interdisciplinary vocabulary. This approach re-incorporates the direct experience of the physical body with a dramaturgical view of its meaning-making performance in relation to its environment at the center of the technologically mediated design process. As such, performative prototyping has the potential to stimulate and accelerate the generation, development, and testing of ideas in the broader field of Collaborative Virtual Environment (CVE) supported HCI design practice.

\section{ACKNOWLEDGMENTS}

I want to thank my teacher colleagues Marloeke van der Vlugt, Lotte Vergouwen, and Stephan Duquesnoy at the HKU University of the Arts Utrecht for sharing their observations and insights concerning the design processes of the two case studies. Furthermore, I'm deeply thankful for the openness, commitment, and creativity of the participating students in exploring and innovating the field of mixed reality performance design, as well as for the generous and supportive NEOS community in sharing their time and know-how in a true open-source manner. This research has been supported through the Doctoral Grant for Teachers of the Dutch Research Council (NWO).

\section{REFERENCES}

[1] Rasmus Vestergaard Andersen, Søren Lekbo, René Engelhardt Hansen, and Lars Elbæk. 2020. Movement-Based design methods: A typology for designers. In Proceedings of the 14th European Conference on Game Based Learning, ECGBL 2020, 637-645. https://doi.org/10.34190/GBL.20.082

[2] Laura Aymerich-Franch. 2018. Is mediated embodiment the response to embodied cognition? New Ideas in Psychology 50: 1-5. https://doi.org/10.1016/j. newideapsych.2018.02.003

[3] Amber Bartosh. 2019. Experimental Applications of Virtual Reality in Design Education. In Ubiquity and Autonomy - Paper Proceedings of the 39th Annual Conference of the Association for Computer Aided Design in Architecture, ACADIA $2019,10$.

[4] Steve Benford and Gabriella Giannachi. 2011. Performing Mixed Reality. The MIT Press, Cambridge, Mass.

[5] Steve Benford, Chris Greenhalgh, Tom Rodden, and James Pycock. 2001. Collaborative virtual environments. Communications of the ACM 44, 7: 79-85. https://doi.org/10.1145/379300.379322

[6] Marion Buchenau and Jane Fulton Suri. 2000. Experience prototyping. In Proceedings of the conference on Designing interactive systems processes, practices, methods, and techniques - DIS '00, 424-433. https://doi.org/10.1145/347642.347802

[7] Paul Cegys and Joris Weijdom. 2020. Mixing realities: reflections on presence and embodiment in intermedial performance design of Blue Hour VR. Theatre and Performance Design 6, 1-2: 81-101. https://doi.org/10.1080/23322551.2020.1785710

[8] Aydin Ciano. 2018. Phenomenological Traditions. In The SAGE Encyclopedia of Communication Research Methods, Mike Allen (ed.). SAGE Publications, Inc, 2455 Teller Road, Thousand Oaks California 91320, 1228-1230. https://doi.org/10.4135/ 9781483381411.n426

[9] Constant foundation. Envision Mixed Reality - Enter New Babylon. Constant 101. Retrieved February 10, 2021 from https://constant101.nl/projects/envisionmixed-reality

[10] Mark R. Costa, Sung Yeun Kim, and Frank Biocca. 2013. Embodiment and Embodied Cognition. In Virtual Augmented and Mixed Reality. Designing and Developing Augmented and Virtual Environments (Lecture Notes in Computer Science), 333342. https://doi.org/10.1007/978-3-642-39405-8_37

[11] N. Dahlbäck, A. Jönsson, and L. Ahrenberg. 1993. Wizard of Oz studies - why and how. Knowledge-Based Systems 6, 4: 258-266. https://doi.org/10.1016/09507051(93)90017-N

[12] Peter Dalsgaard. 2010. Research in and through design: an interaction design research approach. In Proceedings of the 22nd Conference of the Computer-Human Interaction Special Interest Group of Australia on Computer-Human Interaction (OZCHI '10), 200-203. https://doi.org/10.1145/1952222.1952265

[13] Peter Dalsgaard and Lone Koefoed Hansen. 2008. Performing perception - staging aesthetics of interaction. ACM Transactions on Computer-Human Interaction 15, 3: 13:1-13:33. https://doi.org/10.1145/1453152.1453156

[14] Paul Dourish. 2001. Where the action is: the foundations of embodied interaction. MIT Press, Cambridge, Mass.

[15] Steven Dow, Jaemin Lee, Christopher Oezbek, Blair MacIntyre, Jay David Bolter, and Maribeth Gandy. 2005. Wizard of $\mathrm{Oz}$ interfaces for mixed reality applications. In CHI '05 Extended Abstracts on Human Factors in Computing Systems (CHI EA '05), 1339-1342. https://doi.org/10.1145/1056808.1056911

[16] Anthony Frost and Ralph Yarrow. 1989. Improvisation in Drama. Palgrave Macmillan UK, London. https://doi.org/10.1007/978-1-349-20948-4

[17] HKU. fUNK trailer. Retrieved February 10, 2021 from https://youtu.be/fnxHD0_ FIh4

[18] K. Höök. 2018. Designing with the Body: Somaesthetic Interaction Design. MIT Press.

[19] Eva Hornecker. 2011. The role of physicality in tangible and embodied interactions. Interactions 18, 2: 19-23. https://doi.org/10.1145/1925820.1925826

[20] M. Husinsky and F. Bruckner. 2018. Virtual Stage: Interactive Puppeteering in Mixed Reality. In 2018 IEEE 1st Workshop on Animation in Virtual and Augmented Environments (ANIVAE), 1-7. https://doi.org/10.1109/ANIVAE.2018.8587270

[21] Institute for Immersive Learning. VR/AR-Apps, (Collaboration) Working / Learning. Retrieved February 10, 2021 from https://www.immersivelearning.institute/ solution-apps/

[22] Lyn Jeffrey and Toshi Anders Hoo. 2019. Leading-edge behaviors from the world of social VR. Institute for the future. Retrieved from https://www.iftf.org/en/ socialvrleb/

[23] Mina C. Johnson-Glenberg. 2019. The Necessary Nine: Design Principles for Embodied VR and Active Stem Education. In Learning in a Digital World: Perspective on Interactive Technologies for Formal and Informal Education, Paloma Díaz, Andri Ioannou, Kaushal Kumar Bhagat and J. Michael Spector (eds.). Springer, Singapore, 83-112. https://doi.org/10.1007/978-981-13-8265-9_5

[24] Mina C. Johnson-Glenberg, David A. Birchfield, Lisa Tolentino, and Tatyana Koziupa. 2014. Collaborative embodied learning in mixed reality motion-capture environments: Two science studies. Journal of Educational Psychology 106, 1: 86-104. https://doi.org/10.1037/a0034008 
[25] Brigitte Jordan and Austin Henderson. 1995. Interaction Analysis: Foundations and Practice. The fournal of the Learning Sciences 4, 1:39-103.

[26] Stephen Kaplin. 1999. A Puppet Tree: A Model for the Field of Puppet Theatre. TDR/The Drama Review 43 3: 28-35. https://doi.org/10.1162/105420499760347306

[27] Martine Kei Green-Rogers. 2016. What Is Devised Theatre? The Theatre Times. Retrieved July 24, 2021 from https://thetheatretimes.com/what-is-devised-theatre/

[28] Konstantina Kilteni, Raphaela Groten, and Mel Slater. 2012. The Sense of Embodiment in Virtual Reality. Presence: Teleoperators and Virtual Environments 21, 4 373-387. https://doi.org/10.1162/PRES_a_00124

[29] Ji-hye Lee, Lily Díaz-Kommonen, and Yu Xiao. 2019. Applying Embodied Design Improvisation for Physical Interaction in Augmented and Virtual Reality. Archives of Design Research 32, 2: 5-17. https://doi.org/10.15187/adr.2019.05.32.2.5

[30] Junghyo Lee, Junghun Kim, and Jae Young Choi. 2019. The adoption of virtual reality devices: The technology acceptance model integrating enjoyment, social interaction, and strength of the social ties. Telematics and Informatics 39: 37-48 https://doi.org/10.1016/j.tele.2018.12.006

[31] Hans-Thies Lehmann. 2006. Postdramatic Theatre. Routledge, London, UK.

[32] Lian Loke and Thecla Schiphorst. 2018. The somatic turn in human-computer interaction. Interactions 25, 5: 54-5863. https://doi.org/10.1145/3236675

[33] Elena Márquez Segura, Laia Turmo Vidal, Asreen Rostami, and Annika Waern. 2016. Embodied Sketching. In Proceedings of the $2016 \mathrm{CHI}$ Conference on Human Factors in Computing Systems (CHI '16), 6014-6027. https://doi.org/10.1145/ 2858036.2858486

[34] Elena Márquez Segura, Laia Turmo Vidal, and Asreen Rostami. 2016. Bodystorming for Movement-Based Interaction Design. Human Technology 12, 2: 193-251. https://doi.org/10.17011/ht/urn.201611174655

[35] Paul R. Messinger, Eleni Stroulia, Kelly Lyons, Michael Bone, Run H. Niu, Kristen Smirnov, and Stephen Perelgut. 2009. Virtual worlds - past, present, and future: New directions in social computing. Decision Support Systems 47, 3: 204-228. https://doi.org/10.1016/j.dss.2009.02.014

[36] Jason Moore. Alien Rescue - The plot thickens with the metamovie. The MetaMovie. Retrieved February 10, 2021 from https:/www.themetamovie.com/

[37] Kristina Moroz-Lapin. 2009. Role play in HCI studies. In Proceedings of HCI Educators 2009 - playing with our education (HCIED), 64-67. https://doi.org/10. 14236/ewic/HCIED2009.12

[38] Claudia Núñez-Pacheco. 2018. Reflection through Inner Presence: A Sensitising Concept for Design. Multimodal Technologies and Interaction 2, 1: 5. https://doi. org/10.3390/mti2010005
[39] Dennis Schleicher, Peter Jones, and Oksana Kachur. 2010. Bodystorming as embodied designing. Interactions 17, 6: 47-51. https://doi.org/10.1145/1865245.1865256

[40] Andreas Schmeil, Martin Eppler, and Sara Freitas. 2012. A Structured Approach for Designing Collaboration Experiences for Virtual Worlds. Fournal of the Association for Information Systems 13, 10: 836-860. https://doi.org/10.17705/1jais.00309

[41] Lawrence Shapiro and Steven A. Stolz. 2019. Embodied cognition and its significance for education. Theory and Research in Education 17, 1: 19-39. https: //doi.org/10.1177/1477878518822149

[42] Richard Shusterman. 2012. Thinking through the body: essays in somaesthetics. Cambridge University Press, Cambridge, UK; New York.

[43] David Sirkin and Wendy Ju. 2015. Embodied Design Improvisation: A Method to Make Tacit Design Knowledge Explicit and Usable. In Design Thinking Research: Building Innovators, Hasso Plattner, Christoph Meinel and Larry Leifer (eds.). Springer International Publishing, Cham, 195-209. https://doi.org/10.1007/978-3319-06823-7_11

[44] Mel Slater. 2018. Immersion and the illusion of presence in virtual reality. British fournal of Psychology 109, 3: 431-433. https://doi.org/10.1111/bjop.12305

[45] Solirax. 2021. Neos VR Metaverse. Neos VR Metaverse. Retrieved February 10, 2021 from https://neos.com/

[46] Pieter Jan Stappers and Elisa Giaccardi. 2017. Research through Design. In The Encyclopedia of Human-Computer Interaction (2nd ed.), Mads Soegaard and Rikke Friis Dam (eds.). The Interaction Design Foundation, 94. Retrieved from https://www.interaction-design.org/literature/book/the-encyclopedia-ofhuman-computer-interaction-2nd-ed/research-through-design

[47] Vasiliki Tsaknaki, Madeline Balaam, Anna Ståhl, Pedro Sanches, Charles Windlin, Pavel Karpashevich, and Kristina Höök. 2019. Teaching Soma Design. In Proceedings of the 2019 on Designing Interactive Systems Conference (DIS '19), 1237-1249. https://doi.org/10.1145/3322276.3322327

[48] Peng Wang, Peng Wu, Jun Wang, Hung-Lin Chi, and Xiangyu Wang. 2018. A Critical Review of the Use of Virtual Reality in Construction Engineering Education and Training. International fournal of Environmental Research and Public Health 15, 6. https://doi.org/10.3390/ijerph15061204

[49] Danielle Wilde, Anna Vallgårda, and Oscar Tomico. 2017. Embodied Design Ideation Methods: Analysing the Power of Estrangement. In Proceedings of the 2017 CHI Conference on Human Factors in Computing Systems (CHI '17), 5158-5170. https://doi.org/10.1145/3025453.3025873

[50] John Zimmerman, Jodi Forlizzi, and Shelley Evenson. 2007. Research through design as a method for interaction design research in HCI. In Proceedings of the SIGCHI Conference on Human Factors in Computing Systems (CHI '07), 493-502. https://doi.org/10.1145/1240624.1240704 